\title{
Research on refined mesh Scheme of Manifold Element in Adaptive Numerical Manifold Method
}

\author{
Hongfen $\mathrm{Gao}^{1, \mathrm{a}^{*}}$, Gaofeng Wei $\mathrm{i}^{2, \mathrm{~b}}$ \\ ${ }^{1}$ Department of Mechanical Engineering, Shan Dong Polytechnic Jinan, 250104, China \\ ${ }^{2}$ School of Mechanical and Automotive Engineering, Qilu University of Technology, Jinan, 250353, \\ China \\ agaohongfen@126.com, bweigaofeng@126.com
}

\begin{abstract}
Keywords: numerical manifold method; finite cover technology; adaptive analysis; adaptive strategy; mesh refinement

Abstract: Numerical manifold method is a numerical method that widely used in rock mass engineering, its core technology is finite cover of manifold analysis. The manifold element of the numerical manifold method is formed through the finite cover. In this paper the adaptive analysis method based on the manifold unit is studied, and the adaptive strategy of numerical manifold is established, the refined mesh scheme of triangle manifold unit is given. The numerical example shows the effectiveness of the scheme.
\end{abstract}

\section{Introduction}

The numerical manifold method is a modern numerical method, the manifold unit of the numerical manifold method is formed through the finite cover. The method can solve the problems of continuity and discontinuity. The finite cover is composed of mathematical cover and physical mesh, the mathematical cover determines the solution accuracy. Up to now, the basic theory of NMM has received preliminary improvement, but as a numerical method based on grid, the research of the grid problem is still particularly important [1-2].

In much rock mass engineering problem that contains discontinuous deformation, the physical parameters and numerical solution of rock mass are very complicated, which may make the density of the manifold element be correspondingly increased for solving region [3]. If the initial units are refined at the complicated region, the accuracy of numerical solution will be improved, but the amount of calculation will also increase in the case. On the contrary, if we reduce the number of units, the amount of calculation and precision will be dropped. So there is a problem of improving the accuracy and reducing the computing time [4-5].

In order to solving the above problem, the mesh subdivision of the manifold unit is studied and the adaptive strategy is established based on the adaptive grid analysis technology, and the adaptive numerical manifold method is presented. the purpose of this paper is to reduce the time of numerical calculation on the premise of improving accuracy. Finally, the refined mesh scheme of triangle manifold element is discussed.

\section{The adaptive strategy of manifold unit}

For two-dimensional problem of elasticity, mathematical cover of Delaunay triangles and physical grid are used to form manifold element under the physics cover. According to the circumcircle characteristic of Delaunay triangle element, that the characteristic is that any node of Delaunay triangle subdivision cannot fall in any inscribed circle of triangle element, the manifold element is refined based on the Bowyer-Watson algorithm. The basic idea of the Bowyer-Watson algorithm is to determine original manifold element firstly, and then refine manifold element according to self-improved Bowyer-Watson algorithm, the algorithm steps as follows:

(1) Establishing initial Delaunay triangular meshes which can cover the whole solution domain, the solution domain is defined as mathematical mesh, and the mathematical mesh is overlapped on physical grid, the manifold element is formed on the physical cover. 
(2) Manifold element is refined with the improved Bowyer-Watson method (as shown in Fig. 1). An irregular triangle element in manifold unit is selected and a new node neutral is inserted in its longest edge, all the triangles that the circumscribed circle includes $P$ point are found, then these triangles are deleted, and a cavity is formed, $P$ point is connected to each point on the cavity in turn, the new unit is formed after subdivision.

(3) Step 2 can be repeated on the edge of the other coarse triangular element, and the edges having completed the above process are accumulated.

(4) According to the required precision of researched problem, repeating the above steps for corresponding triangular elements in the manifold method, refined step is stopped until it meets the accuracy requirement.

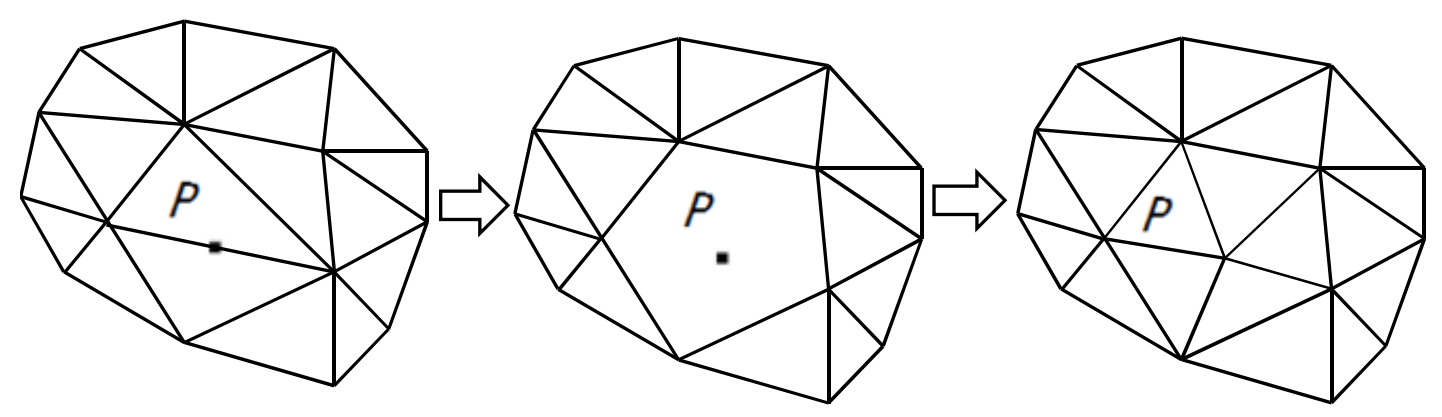

Fig.1. The refined process of triangle manifold element

\section{The judgment of manifold element}

When a midpoint is inserted in a manifold element of target area, we need to judge the manifold element, and size function can be used in the method.

The magnitude of size function in a certain physical cover is called size of physical cover, if the size of an element meets the requirements, the element is called a good element, on the other hand, it is called a bad element. The distinction between good and bad elements have different evaluation standard. In this paper the evaluation standard is the ratio of the radius of circumscribed circle $r(i)$ and the size of external center of circle $d(i)$ in manifold element. If $(r(i)) /(d(i)) \leq \delta(\delta$ is a constant, usually $\delta=1.0-1.5$ ), then the element is a good element, or it is a bad element. The size of $\delta$ is an empirical value, it has changed according to the different situation of manifold element. So we always choose the bad element to add point when we add point in the manifold element.

\section{The size function of grid}

The size function of element is related to the density of element point, so it must be given reasonably. In this paper the method of generating size function is divided into two steps.

The first step, the initial mathematical mesh is generated. Assuming there have existed the initial Delaunay triangle elements that are generated by Bowyer-Watson method in the solved area. The previously defined discrete point with special size requirement is inserted into the initial Delaunay triangle element after generating the initial Delaunay triangular element by boundary point in the solved area, the solved area that contains special discrete points has the big triangles that are determined by a number of vertices size. According to the size of the vertices, a series of points in big triangle are generated, namely mathematical mesh, its size is gradual. The size of mathematical grid point can reflect the variety regulation of the size of the required element, so we just need to storage the information of mathematical grid point without connecting it into an element.

The second step, the size function need to calculate through the grid size formed by physical covers. Relative to the manifold element, physical cover is independent and fixed. It no changes after generated. A new size function of manifold element can be got after inserting the node to generate new element. 


\section{Numerical Example}

The geometry of numerical example is shown as Fig.2(a), Fig.2(a) is original graph that is not meshed, the refined scheme described in this paper is used to refine the example, Fig. 2(b) is the initial divisive grids, Figs. 2 (c) - 2 (g) are the refined grids.

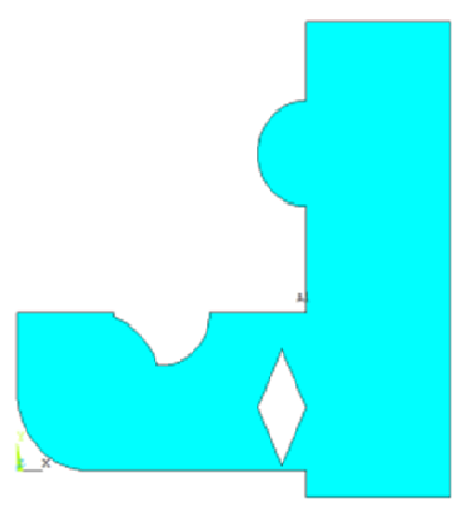

2(a). the initial graph

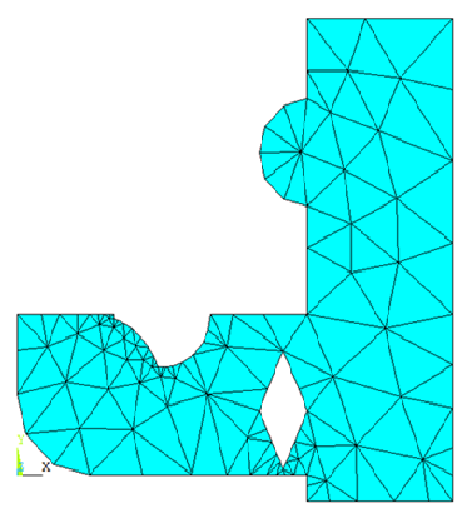

2(c). the first refinement

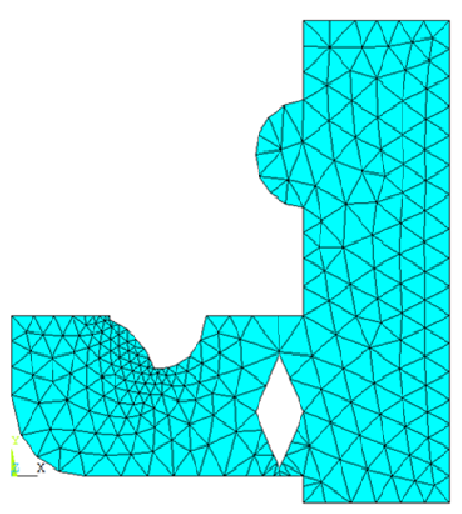

2(e). the third refinement

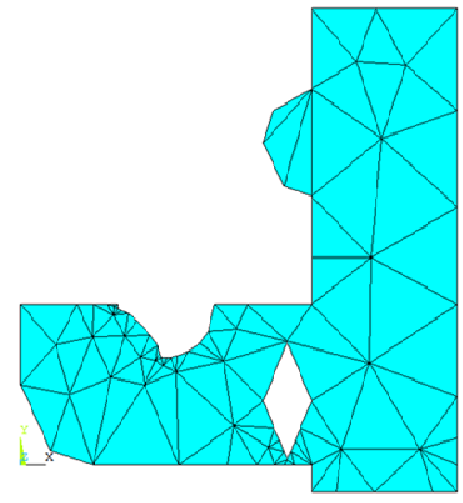

2(b). the initial grid

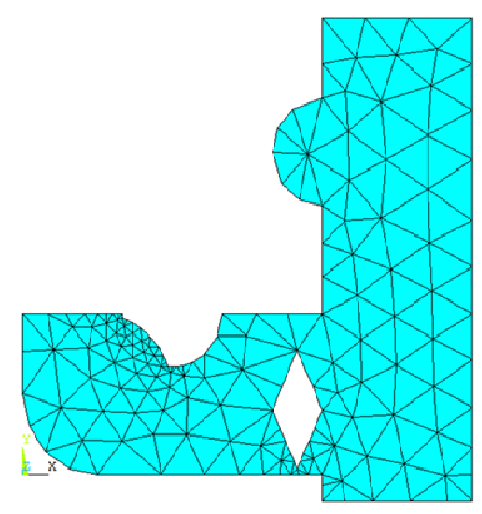

2(d). the second refinement

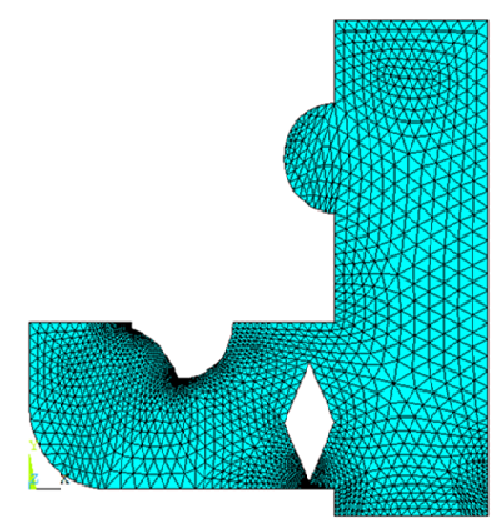

2(f). the fourth refinement 


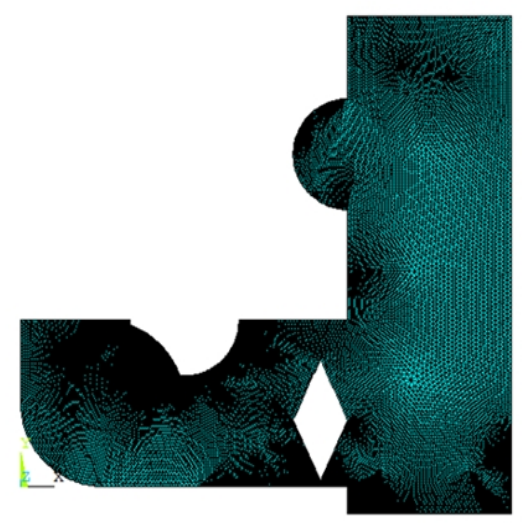

$2(\mathrm{~g})$. the fifth refinement

Fig.2. The initial and the refined meshes

There are some inner angle areas in the numerical example, it is well known that the stress concentration occurs in the inner angle areas for the elastic problems, so it is particularly important to refine the local interior area. The refined scheme of triangle element proposed in this paper can reduce the number of singular triangle elements in the case of initial triangle element is more sparse. The shape of element can be close to the regular triangle, the quality of manifold element is more and more high with the increase of refined number. The manifold elements, which meet the precision requirement in the stress concentration area, can be obtained, and the precision of numerical calculation can be improved

\section{Conclusions}

The adaptive manifold element is analyzed, and the adaptive strategy of the numerical manifold method and encrypted the triangle manifold element are put forward in this paper. The numerical results show that the quality of manifold element has greatly improved through the adaptive analysis. This method has good applicability, and can be applied to practical engineering problems of rock mass.

\section{Acknowledgement}

The work is supported by the National Natural Science Foundation of China through grant 11271234 and the Science Program of Shandong Provincial Education Department through grant J13LJ51.

\section{References}

[1] X.W.Zhang, Z.R.Zhang, W.G.Lv. Numerical manifold method research and application progress[J]. Progress in mechanics, 40 (2010) 1-12.

[2] H.F.Gao, G.F.Wei. Stress intensity factor for interface cracks in bimaterials using complex variable meshless manifold method[J]. Mathematical Problems in Engineering, 1(2014) 1-8.

[3] K.Terada, A.Maruyama, M.Kurumatani. Eulerian finite cover method for quasi-static equilibrium problems of hyperelastic bodies[J]. Numer. Methods Eng., 23(2007) 1081-1094.

[4] Li Shuchen, Cheng Yumin. Meshless numerical manifold method based on unit partition[J]. Acta Mechanica Sinica, 36(2004) 496-500.

[5] Jeen-Shang Lin, A mesh-based Partition of Unity Method for Discontinuity Modeling[J]. Computer Methods and Applied Mechanics in Engineering, 192 (2003) 1515-1532 